\title{
EDITORIAL
}

\section{Comprehensive state-of-the-art overview of neonatologist performed echocardiography: Steps towards standardization of the use of echocardiography in neonatal intensive care}

\author{
Pediatric Research (2018) 84:472-473; \\ https://doi.org/10.1038/s41390-018-0118-5
}

In recent years, the interest in the use of echocardiography to assess critically ill premature and term newborns has exponentially increased. Neonatologist performed echocardiography (NPE) has the potential to enhance clinical hemodynamic assessment by adding important physiologic information that can guide hemodynamic management. This can ultimately improve outcome in this vulnerable population. However, propitious impact on patient care with the use of NPE can only be accomplished by following two important guiding principles: (1) competence: to ensure a high standard of image quality and interpretation of the findings and (2) logic and reason: where the findings are used in the context of the clinical scenario. This requires a structured training program in NPE skills, as well as technical and clinical guidelines for the use of NPE.

The special interest group "Neonatologist Performed Echocardiography Collaborative" is an initiative of the "Circulation, Oxygen Transport \& Haematology" Section of the European Society for Pediatric Research (ESPR). The group was convened in 2014 with the goal of promoting the responsible use of echocardiography by neonatologists to enhance clinical care of newborn infants by formulating "state-of-the art" guidelines. Currently, the NPE group consists of 38 members originating from 17 countries worldwide with specific expertize in NPE. In 2016, a consensus statement was published by this group with recommendations for NPE training and accreditation in Europe. ${ }^{1}$ Subsequently the group developed comprehensive technical and clinical reviews encompassing many aspects of the use of NPE in the neonatal intensive care unit (NICU). These review papers are presented together in a dedicated online, open-access supplement to this journal. The first review is an introduction to NPE describing the indications for echocardiography in the neonatal setting, explaining the basic physics of ultrasound and presenting a structured approach to image acquisition. ${ }^{2}$ The second paper briefly describes the current training recommendations across several jurisdictions including Europe, North America, and Australia, outlines the steps required to achieve formal accreditation of NPE practice in Europe, and outlines the foundation for building a sustainable governance structure. ${ }^{3}$ Two subsequent reviews focus on the emerging field of newer echocardiographic functional assessment including tissue Doppler velocity imaging (TDI), event timing, ${ }^{4}$ deformation imaging, and rotational mechanics. ${ }^{5}$ Those reviews provide guidance to image acquisition, measurement, interpretation and reference values for those newer modalities. Four clinically oriented reviews describing the role of NPE in the assessment and management of persistent patent ductus arteriosus $\left(\mathrm{PDA}^{6}\right)$, neonatal shock, ${ }^{7}$ persistent pulmonary hypertension of the newborn $\left(\mathrm{PPHN}^{8}\right)$, and neonatal heart failure, ${ }^{9}$ complete the comprehensive overview of the use of NPE in neonatal care in this supplement.

The next objective of the NPE group is to implement a panEuropean governance structure with the responsibility to develop a certification and accreditation program and set standards for competency and excellence in NPE under the umbrella of the European Board of Neonatology (EBN) within the ESPR. Moreover, NPE will be incorporated into the next, revised version of the European Curriculum and Assessment Framework for Subspecialty Training in Neonatology. The ESPR and EBN will give their full support to help this collaborative to unify NPE practice throughout Europe at the highest, commonly agreed standards, which will be in the benefit to the patients and their families. Close collaboration with our colleagues in pediatric cardiology is pivotal and their full endorsement is paramount. For this, every effort is being made to achieve complete appreciation of this NPE initiative and it proposed governance structure by societies of pediatric cardiology and pediatric echocardiography. The overall aim of this endeavor is to ensure the safe application of echocardiography in the clinical setting to enhance hemodynamic assessment, improve our management strategies, and foster collaborative research.

\section{ADDITIONAL INFORMATION}

Competing interests: The authors declare no competing interests.

Publisher's note: Springer Nature remains neutral with regard to jurisdictional claims in published maps and institutional affiliations.

W. P. de Boode ${ }^{1}$, C. C. Roehr ${ }^{2}$ and A. El-Khuffash ${ }^{3,4}$ ${ }^{1}$ Department of Neonatology, Radboud University Medical Center, Radboud Institute for Health Sciences, Amalia Children's Hospital, Nijmegen, The Netherlands; ${ }^{2}$ Department of Paediatrics, University of Oxford, John Radcliffe Hospital, Oxford, UK; ${ }^{3}$ Department of Neonatology, The Rotunda Hospital, Dublin, Ireland and ${ }^{4}$ Department of Pediatrics, The Royal College of Surgeons, Dublin, Ireland

Correspondence: W. Boode (willem.deboode@radboudumc.nl)

\section{REFERENCES}

1. de Boode, W. P. et al. Recommendations for neonatologist performed echocardiography in Europe: consensus statement endorsed by European Society for Paediatric Research (ESPR) and European Society for Neonatology (ESN). Pediatr. Res. 80, 465-471 (2016).

2. Groves, A. M., et al. Introduction to Neonatologist Performed Echocardiography. Pediatr. Res. (2018). https://doi.org/10.1038/s41390-018-0076-y.

3. Singh, Y., et al. Education, training and accreditation of neonatologist performed echocardiography in Europe - framework for practice. Pediatr. Res. (2018). https:// doi.org/10.1038/s41390-018-0078-9.

4. Nestaas, E., Schubert, U., de Boode, W. P., EL-Khuffash, A. Tissue doppler velocity imaging and event timings in neonates. A guide to image acquisition, measure- 
ment, interpretation and reference values. Pediatr. Res. (2018). https://doi.org/ 10.1038/s41390-018-0079-8.

5. EL-Khuffash, A., Schubert, U., Levy, P. T., Nestaas, E., de Boode, W. P. Deformation imaging and rotational mechanics in neonates: a guide to image acquisition, measurement, interpretation and reference values. Pediatr. Res. (2018). https://doi. org/10.1038/s41390-018-0080-2.

6. Van Laere, D., et al. Application of neonatologist performed echocardiography in the management of a patent ductus arteriosus. Pediatr. Res. (2018). https://doi.org/ 10.1038/s41390-018-0082-0.
7. de Boode, W. P., et al. The role of neonatologist performed echocardiography (NPE) in the assessment and management of neonatal shock. Pediatr. Res. (2018). https://doi.org/10.1038/s41390-018-0077-x.

8. de Boode, W. P., et al. Application of neonatologist performed echocardiography in the assessment and management of persistent pulmonary hypertension of the newborn. Pediatr. Res. (2018). https://doi.org/10.1038/s41390-018-0075-z.

9. Levy, P. T., et al. Application of neonatologist performed echocardiography in the assessment and management of neonatal heart failure unrelated to congenital heart disease. Pediatr. Res. (2018). https://doi.org/10.1038/s41390-018-0081-1. 